\title{
A Modeling Method for Small Packing Particles in Electromagnetic Simulation
}

\author{
Yi Cheng Zhong, Yu Jian Cheng, and Yong Fan \\ Fundamental Science on Extreme High Frequency Laboratory, School of Electronic Engineering, \\ University of Electronic Science and Technology of China, Chengdu 611731, China
}

Correspondence should be addressed to Yu Jian Cheng; chengyujian@uestc.edu.cn

Received 28 August 2015; Revised 12 February 2016; Accepted 29 February 2016

Academic Editor: Luciano Tarricone

Copyright (C) 2016 Yi Cheng Zhong et al. This is an open access article distributed under the Creative Commons Attribution License, which permits unrestricted use, distribution, and reproduction in any medium, provided the original work is properly cited.

\begin{abstract}
A modeling method for small packing particles is proposed in this paper, especially for the application of the electromagnetic simulation. This method is able to model random distributed and mutual-contacted packing particles efficiently and accurately. This modeling method is faster than a commonly used software, especially for large number of particles. The accuracy of this modeling method is validated by experiments of packing density and effective permittivity. The established model can be easily imported into commercial electromagnetic simulation software.
\end{abstract}

\section{Introduction}

Most natural substances, such as building materials and minerals, present in the mixed state, especially in the state of packing particles. The propagation and reflection properties of the electromagnetic wave in inhomogeneous materials have been widely studied $[1,2]$. The model of packing particles plays an important role in the research of the groundpenetrating radar, the microwave heating, the microwave desulfurization, and so forth. Therefore, there is an urgent need for building an accurate model of packing particles to study the propagation characteristics of the electromagnetic wave in nature [3-5].

The particle in this paper is defined as the smallest unit of substances with identical electromagnetic properties. Electromagnetic properties of the individual particle are usually easy to recognize by experiment [6-8]. However, when the material is formed by a number of solid packing particles, it is difficult to investigate electromagnetic properties of this mixed-particle material because of the large number and random distribution of these particles [9].

Previous researchers have done rigorous and impressive job on this issue and proposed different methods. Effective complex permittivity of dielectric mixtures can be evaluated by different formulations and rules from theoretical models and experiments [10-14]. However, the theoretical model can only be used in the particles with simple and regular shapes. Besides, the experimental method is affected by the random distribution of particles, and most of the time, the accurate formulations of mixtures cannot be gotten from the experimental results.

Many numerical studies have also been performed to analyze the effective permittivity of dielectric mixtures [1517]. The dynamical solution method (FDTD) is firstly applied to the two-dimensional dielectric mixture [17]. This method is also used in the three-dimensional analysis in this paper. Besides, there are some other kinds of modeling software, such as PFC 3D, used in fields outside of the electromagnetism [18-23]. They are employed in the model of packing particles in architecture and civil engineering. Their principle is to calculate movements of the particles through boundary conditions and physical properties until a stable state is achieved. The available shapes of particles are only the sphere and the combination of several spheres. Thus, particles with complex shapes are difficult to be modeled. The modeling software also needs a long time as well as a lot of computing resources for the modeling of a large number of particles.

This paper aims to establish an accurate model through a simple and quick process to simulate various packing particles, such as sand, soil, and coal powder. Furthermore, 
the model can be imported into some kinds of commercial electromagnetic simulation software easily. Currently, there are a variety of methods for modeling stacked particles in the region of architecture, mechanics, and geophysics. But very few of them are proper to the electromagnetic research and none of them can be imported into simulation software directly.

This paper will focus on how to model contacted balls and regular polyhedrons to simulate the packing particles. It can also be employed in the modeling of packing particles with complex shapes. The accuracy of the model is validated by experiments of packing density and effective permittivity.

\section{Modeling}

2.1. Model of Packing Spheres. The completed modeling process is shown in Figure 1. The first step is to distribute balls uniformly within the desired region. Supposing the desired region with a length of $L$, a width of $S$, and a height of $H$, the number of spheres, $W$, can be calculated by floor $[(L \times S \times H \times 2) /(a+b)]$. Floor is the function of the rounding down. The diameter range of spheres is $(a, b)$. Secondly, centers of spheres are moved and radii of spheres are gradually increased to make them contact each other. Finally, the modeling will be ended if all the spheres contact at least two other spheres or the edge of the analyzed region. When the process is over, the packing density of the spheres becomes relatively stable and within the possible region validated by experiments [24]. Thus, this model is considered to be consistent with the actual distribution of the packing spheres.

The modeling method is different from these rigorous modeling and analytical methods in architecture and civil engineering. That is because it does not consider the gravity and friction of the balls. However, the modeling method is particularly used for the electromagnetic analysis of relatively small packing particles; thus it can be accepted without the gravity and the friction in the modeling process. Besides, in the electromagnetic simulation of accumulated particles, the more important influence factors are the shape, size, and packing density of the investigated material [16]. The effects of the gravity and the friction are small, which are usually excluded in the analysis of electromagnetic properties of packing particles.

Figure 2 demonstrates some key steps in the modeling process with different cycle time, $P$. The present modeling was carried out with balls, which have diameters ranging in $8 \sim 10 \mathrm{~mm}$, within a cubic region of $75 \times 75 \times 105 \mathrm{~mm}^{3}$. The total modeling time is limited in 10 seconds.

In the electromagnetic analysis of packing particles, the shape, size, and packing density are the most important parameters. The shape and size of particles in the model should be consistent with the actual situation. The criterion for the reasonable modeling is that the packing density of the model is in good agreement with the experimental result.

The experimental result on the packing density of steel balls in cylindrical containers is published by Scott [24]. The relationship between the packing density and the diameter

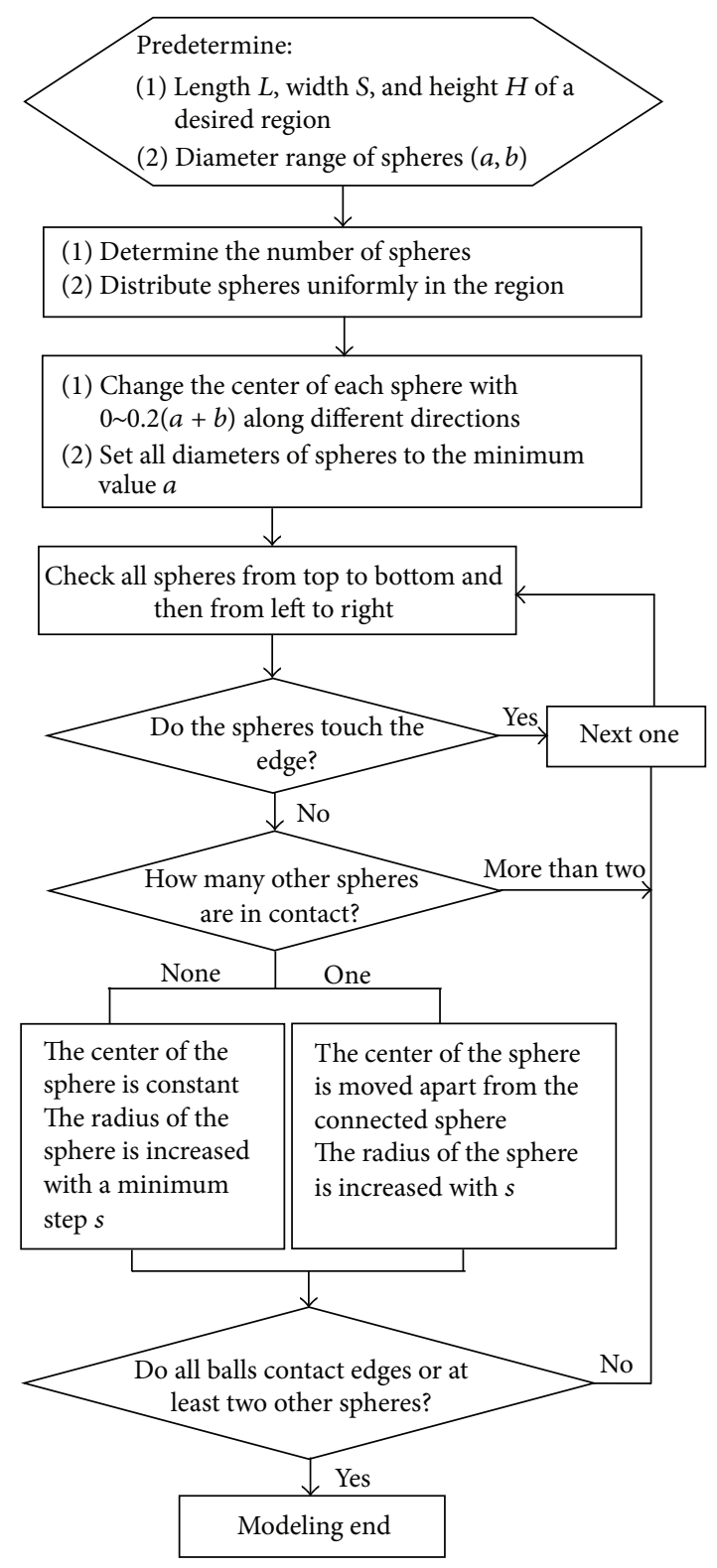

FIGURE 1: Modeling process.

of the cylindrical container is investigated by experiment. The packing density of steel balls is from 0.56 to 0.6 in the cylindrical container. The diameter of the cylindrical container is 10.5 times compared with the diameter of the steel ball. When the container becomes bigger, the packing density will rise to $0.58 \sim 0.62$.

The packing density of the modeling method proposed in this paper should comply with the experimental result. Several models are built in the same condition as described in [24] in order to validate the correctness of our method. The results are shown in Figure 3. Modeling is defined within a cylinder with a diameter of $105 \mathrm{~mm}$ and a height of $120 \mathrm{~mm}$. The diameter of balls is limited in the range of $9 \sim 11 \mathrm{~mm}$ and $4.5 \sim 5.5 \mathrm{~mm}$, respectively. This is because the packing density in a limited container is related to the size of balls. 


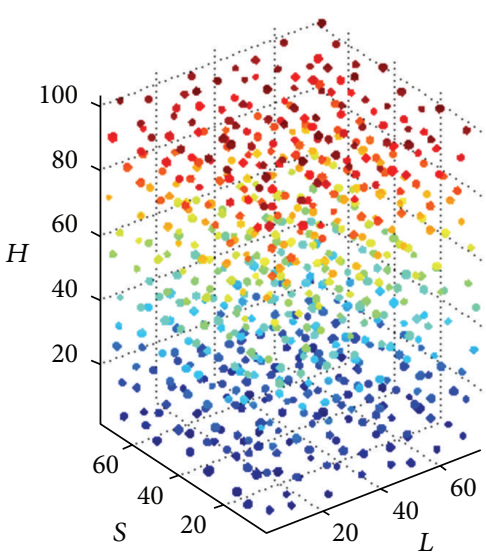

(a)

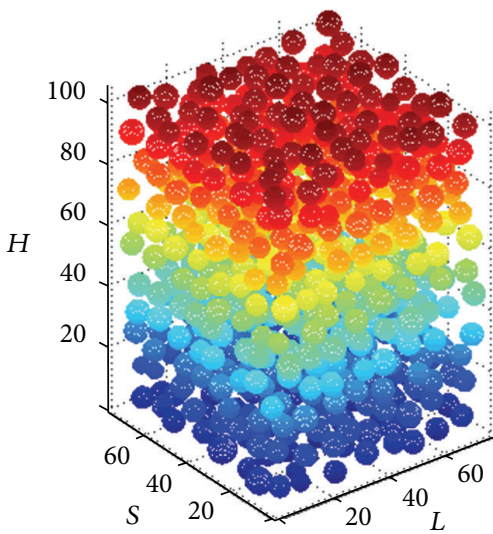

(c)

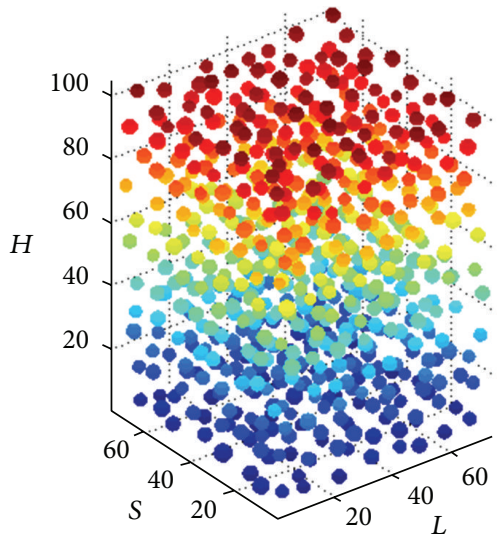

(b)

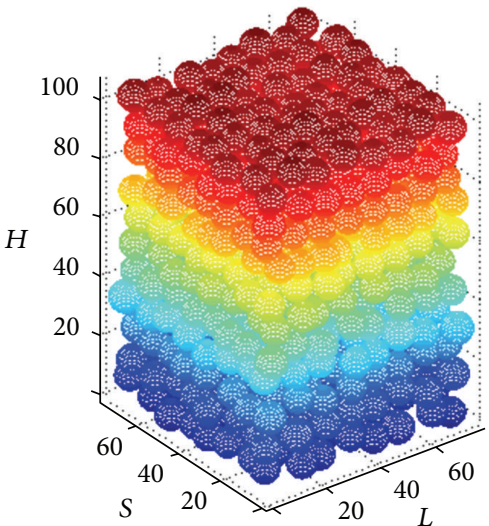

(d)

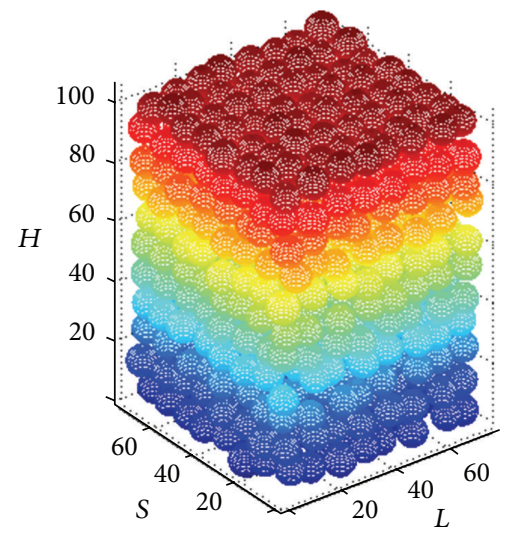

(e)

Figure 2: Modeling process with different cycle times (mm). (a) $P=1$; (b) $P=20$; (c) $P=40$; (d) $P=50$; and (e) $P=55$.

Figure 3 shows a good agreement of the packing density between the modeling result and the experimental result in [24]. Modeling has been implemented 30 times in these two cylindrical containers. Most packing density of models agrees with the result described in [24]. The average packing density of 30 models is 0.585 and 0.601 , respectively. They are nearly middle values in the packing density range. These indicate the stability and reliability of the modeling method.

There are some other modeling methods applied in other areas $[25,26]$, such as PFC 3D. Figure 4 shows the time to model packing balls by use of our method and PFC 3D. Both of the modeling methods are carried out with balls having $8 \sim 10 \mathrm{~mm}$ diameter in the cube having the bottom area of 75 $\times 75 \mathrm{~mm}$. The density of balls is defined as $2000 \mathrm{~kg} / \mathrm{m}^{3}$ and the stiffness of all balls and boundary is $10^{8} \mathrm{~N} / \mathrm{m}$. The friction between balls is set as 1 . The modeling time is defined to be from the beginning of the modeling to reach a steady state. The comparison shows that the proposed method has much less modeling time, which is more and more obvious with the increase of the number of balls. 


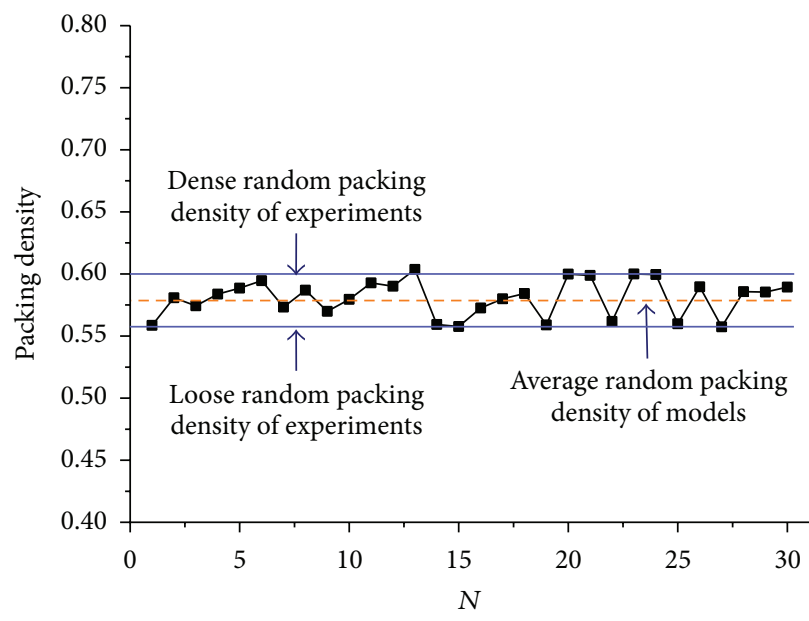

(a)

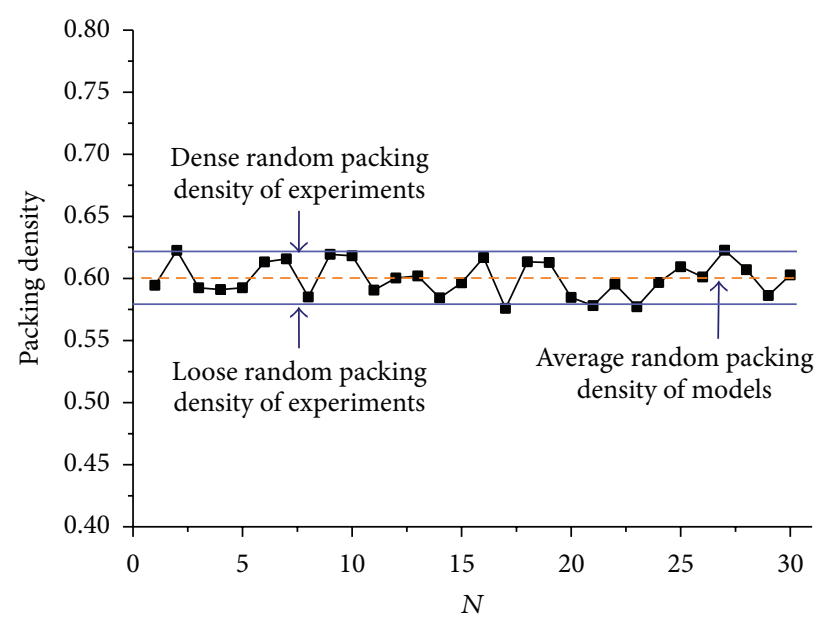

(b)

Figure 3: Comparison of the packing density between the model and the experiment. $N$ is the modeling number. (a) Diameter of balls ranging from $9 \mathrm{~mm}$ to $11 \mathrm{~mm}$. (b) Diameter of balls ranging from $4.5 \mathrm{~mm}$ to $5.5 \mathrm{~mm}$.

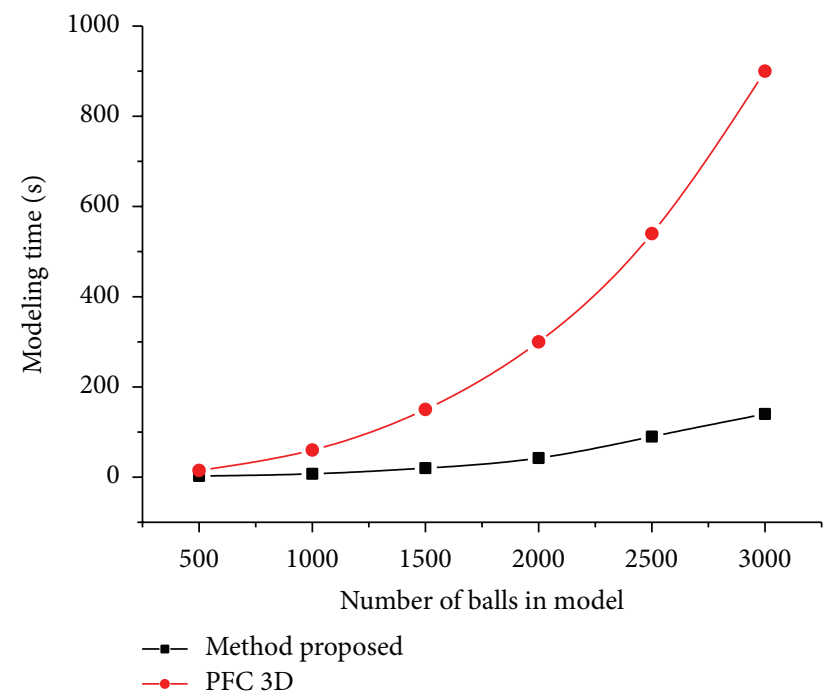

Figure 4: Comparison of the modeling time between PFC 3D and the method proposed in this paper.

2.2. Model of Packing Polyhedrons. Generally speaking, the particles are not of the spherical type in most modeling cases. In this modeling method, spheres can be conveniently converted to polyhedrons.

As an example, these spheres are deformed into polyhedrons for the modeling of packing fragmented FR4 substrate. The shape of particles close to reality can increase the accuracy of the following electromagnetic analysis.

As shown in Figure 5, the first step is to choose the edge number, $N$, of polygons according to the shape of the modeling particle. Then, the balls are divided uniformly by $N$ polygons with $N$ edges. Finally, all the polygons are connected to constitute a polyhedron. This method can transform the spheres into polyhedrons in terms of $N$, which is good for the modeling of some ball-like particles.
This model is built in Matlab software according to the modeling process. The model can be imported into ANSYS HFSS by use of HFSS-MATLAB-Scripting-API directly. Now, the simulation can be started in HFSS.

\section{Correctness Validation of Modeling Method}

To verify the stability and reliability of our modeling method, the model of packing fragmented FR4 as shown in Figure 6 is imported into HFSS and then simulated. The simulated and experimental results will be compared. The investigated frequency band is from $10 \mathrm{GHz}$ to $15 \mathrm{GHz}$. For a FR4 dielectric substrate, the relative permittivity, $\varepsilon_{r}$, approximately linearly decreases from 4.29 to 4.21 within the interested frequency band, while the loss tangent, $\tan \delta$, approximately linearly increases from 0.020 to 0.024 .

The particles of FR4 are filled into a standard rectangular waveguide $(19.050 \mathrm{~mm} \times 9.525 \mathrm{~mm})$ to test the $S$ parameters [11]. The equivalent $\varepsilon_{r}$ and $\tan \delta$ of the packing particles can be calculated from the $S$ parameters and compared with the results obtained by HFSS. The fragmented FR4 is cut off from a $1 \mathrm{~mm}$ thick FR4 substrate with the size of $1 \times 1 \mathrm{~mm}^{2}$. Then, these cube particles are mutually extruded and rubbed to convert them to the ball-like polyhedrons with the diameter of mainly $1.2 \sim 1.5 \mathrm{~mm}$.

First, three famous mixing rules about the effective permittivity will be introduced and their results will be compared with simulations and experiments. The Looyenga model $[27,28]$, which is widely used to calculate $\varepsilon_{r}$ of random distributed balls, is

$$
\sqrt[3]{\varepsilon_{m}}=v_{1} \sqrt[3]{\varepsilon_{1}}+v_{2} \sqrt[3]{\varepsilon_{2}}
$$

The Maxwell-Garnett (MG) mixing rule [29] is

$$
\frac{\varepsilon_{m}-\varepsilon_{2}}{\varepsilon_{m}-2 \varepsilon_{2}}=v_{1} \frac{\varepsilon_{1}-\varepsilon_{2}}{\varepsilon_{1}+2 \varepsilon_{2}} .
$$




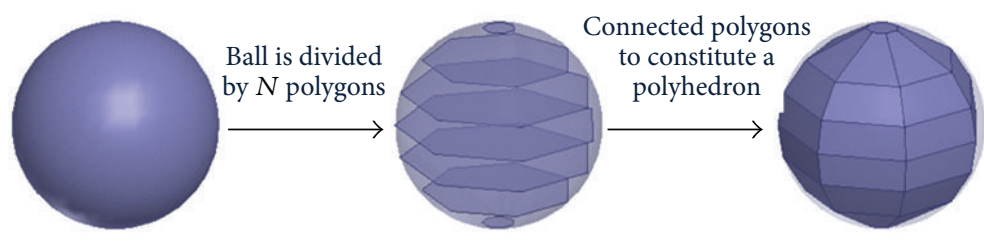

FIGURE 5: Change spheres into polyhedrons.

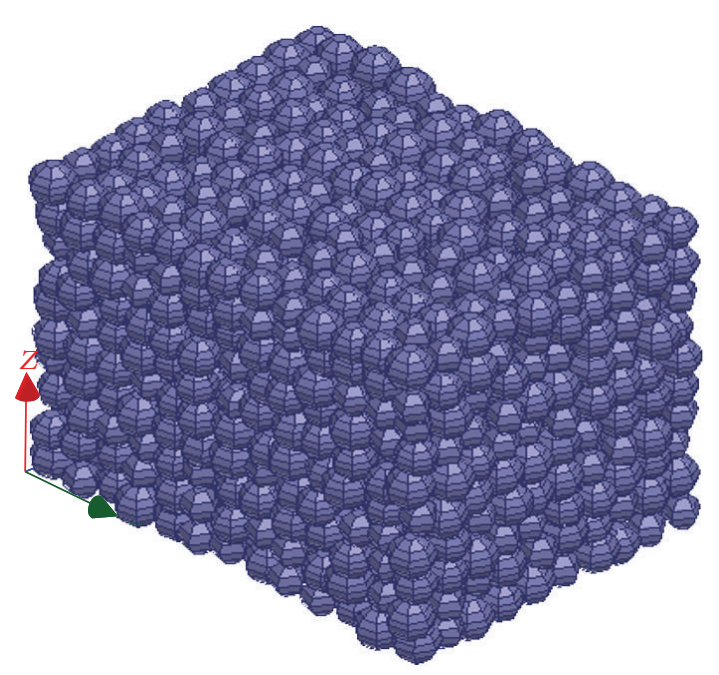

FIgURE 6: Accumulation of polyhedrons.

The Bruggeman mixing rule [30] is

$$
v_{1} \frac{\varepsilon_{1}-\varepsilon_{m}}{\varepsilon_{1}+2 \varepsilon_{m}}=-v_{2} \frac{\varepsilon_{2}-\varepsilon_{m}}{\varepsilon_{2}+2 \varepsilon_{m}},
$$

where $\varepsilon_{m}$ is the equivalent dielectric constant of the packing particles and $v_{1}$ and $v_{2}$ are the volume ratios of the particles and the homogeneous environment, respectively. The volume ratios are calculated by the total volume and the weight of the packing particles. The theoretical $\varepsilon_{m}$ of packing FR4 particles packing in the air $\left(v_{1}=0.58, \varepsilon_{1}=4.21 \sim 4.29 ; v_{2}=0.42, \varepsilon_{2}=1\right)$ of three famous mixing rules is shown in Figure 9.

The measured packing particles in the rectangular waveguide are shown in Figure 7. The vector network analyzer Agilent N5244A is employed to test the $S$ parameters of the waveguide filled with the FR4 particles. Each end of the waveguide is connected to a coaxial converter as shown in Figure 8. A transparent plastic paper with the thickness of $0.08 \mathrm{~mm}$ is clipped between the waveguide and the converter in order to confine the particles in the waveguide.

The ambient temperature is $25^{\circ} \mathrm{C}$ and the relative humidity is $65 \%$ in the laboratory. The packing depth, $H$, should be longer than $0.1 \lambda_{g}\left(\lambda_{g}\right.$ is the guide wavelength). Otherwise, the measured result is inaccurate. Besides, $H$ should not be the integer times of $\lambda_{g} / 2$ because the resonant effect in the waveguide will seriously affect the measured result $[6,10]$. In the testing frequency band, the range of $\lambda_{g}$ is $14.72 \mathrm{~mm} \sim$ $30.48 \mathrm{~mm}$. Thus, the effective range of $H$ is selected as 3 $10 \mathrm{~mm}$ in the measurement.

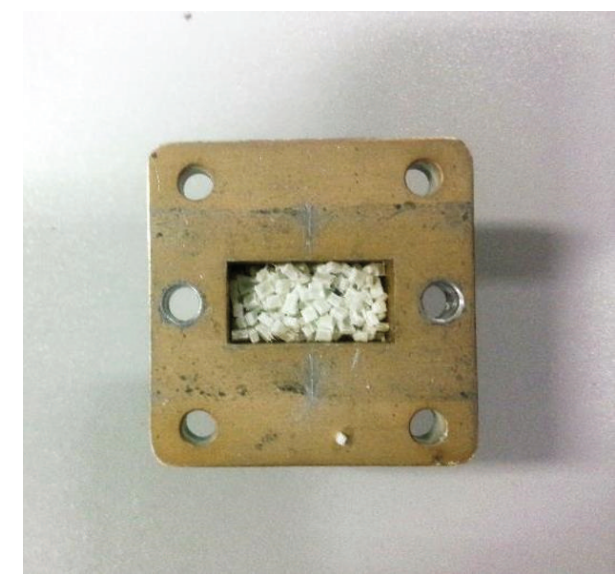

FIGURE 7: FR4 particles in a rectangular waveguide.

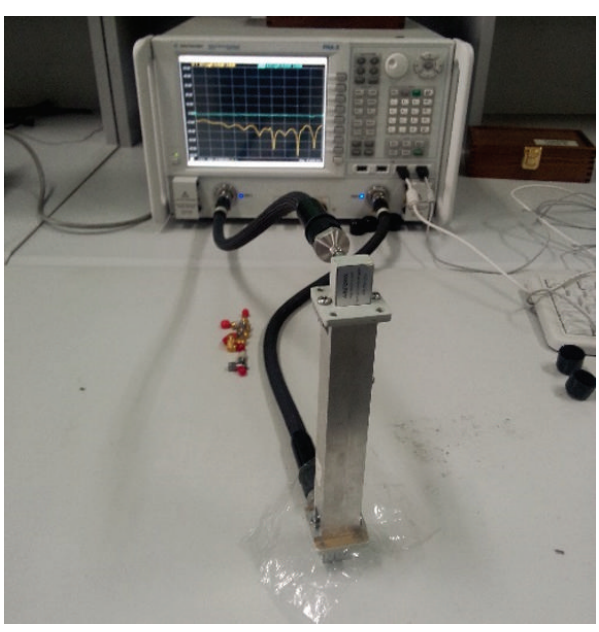

FIGURE 8: Measurement of electromagnetic properties of packing particles in the rectangular waveguide.

The theoretical, experimental, and simulated results of $\varepsilon_{m}$ and $\tan \delta_{m}$ calculated from $S$ parameters $[6,31,32]$ are shown in Figures 9 and 10. These results are the average value of 30 repeated simulations and experiments. In Figure 9, the simulated results of the effective permittivity show good agreement with experimental data in the whole band at $H=$ $3 \mathrm{~mm}$. The MG mixing rule agrees well with the experimental results of the packing polyhedrons with smaller packing depth $H$. In Figure 10, the simulated and experimental effective permittivities still agree well especially in the range of large packing depth, and both increase with the packing 


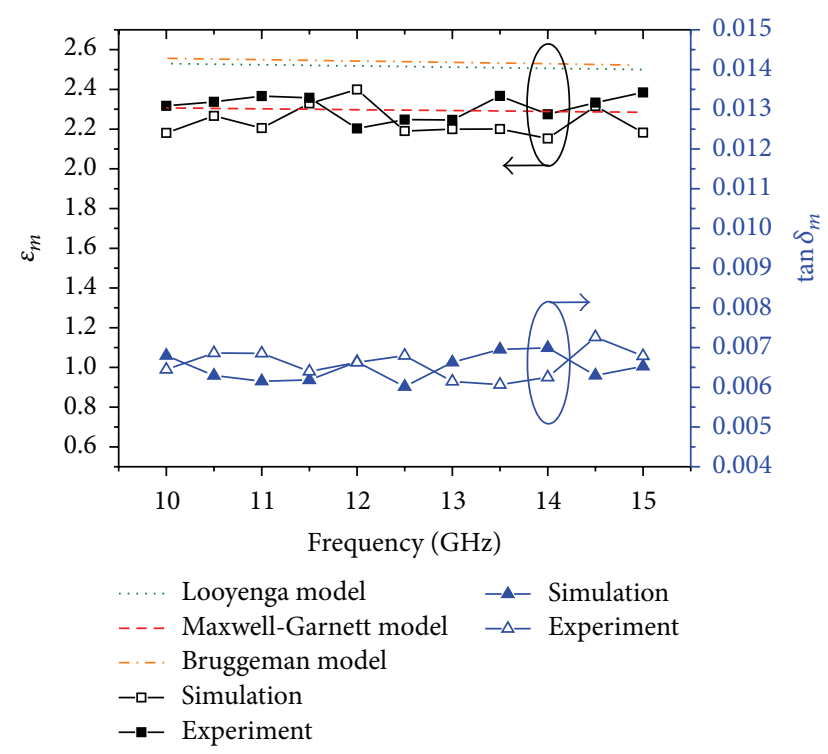

Figure 9: Theoretical, experimental, and simulated results of $\varepsilon_{m}$ and $\tan \delta_{m}$ in the frequency band with $H=3 \mathrm{~mm}$.

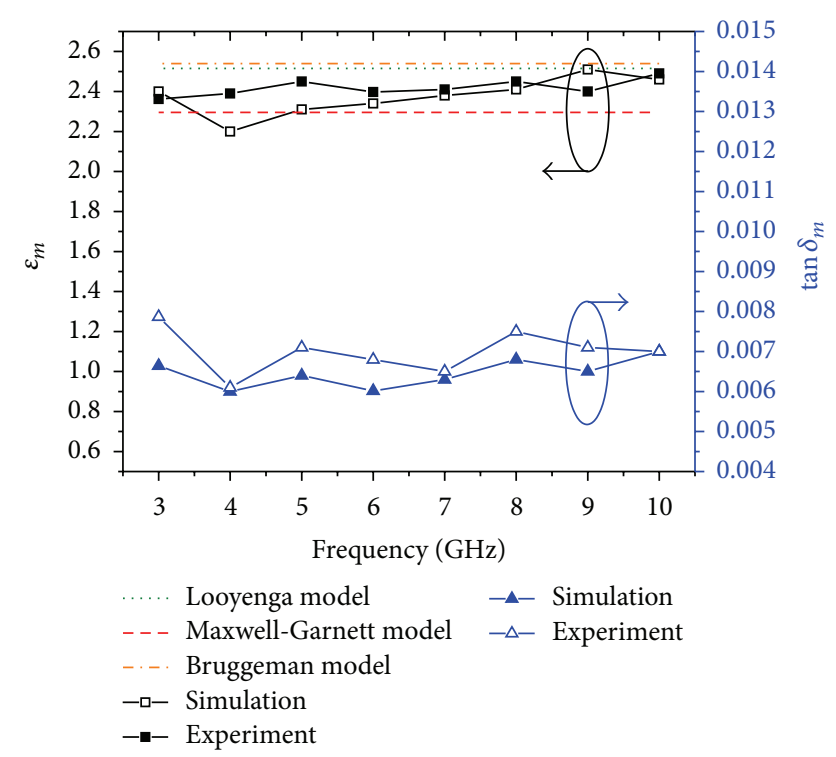

FIgURE 10: Theoretical, experimental, and simulated results of $\varepsilon_{m}$ and $\tan \delta_{m}$ at the center frequency, $12.5 \mathrm{GHz}$, with different packing depth $H$ of the particles.

depth. This is because the random distribution of particles has a significant effect on the result in the case of fewer particles. With the increase of $H$, the difference between the simulated and experimental results becomes smaller. Both of them get closer to the results of the Looyenga rule and the Bruggeman rule. They still fall between the predictions of the MG rule and the Bruggeman rule, which is consistent with the result in [17] for two-dimensional dielectric mixture by using the FDTD method similar to this paper. It is worth pointing out that the simulated and experimental results satisfy the Wiener bounds and the Hashin-Shtrikman bounds as well, since they are often looser than the bound between the MG rule and the Bruggeman rule. The influence of the random distribution of particles will be weakened with the increasing of number of packing particles.

The advantages of modeling and numerical simulation are embodied in the analysis above. Compared with the empirical formula, it takes more conditions into consideration such as shape, size and distribution of the particles, the thickness of packing, and the frequency. Besides, the modeling and simulation seem to be helpful to select the suitable formulation for the effective permittivity. For example, the MG mixing rule shows better agreement with the simulated and experimental results than the other two rules in the analysis of the packing polyhedrons with smaller packing depth $H$.

\section{Conclusion}

A modeling method for the packing particles is proposed in this paper. The packing particles in the shape of sphere or sphere-like polygon can be modeled easily and imported into the electromagnetic simulation software directly. It is much faster than PFC 3D to construct a model with a number of packing balls. It has been verified by experiment and agrees well with formulations and rules of the mixture in literatures. Besides, it is more accurate than the empirical formula to recognize equivalent electromagnetic properties of packing particles due to the fact that more conditions are considered. The modeling method has unique advantages in the field of electromagnetic simulation of packing particles.

\section{Competing Interests}

The authors declare that there are no competing interests regarding the publication of this paper.

\section{Acknowledgments}

This work is supported in part by National Basic Research Program of China under Grant no. 2012CB214900, in part by Program for New Century Excellent Talents in University under Grant no. NCET-13-0089, and in part by National Program for Support of Top-Notch Young Professionals.

\section{References}

[1] H. Looyenga, "Dielectric constants of heterogeneous mixtures," Physica, vol. 31, no. 3, pp. 401-406, 1965.

[2] J. C. Santamarina, A. Klein, and M. A. Fam, "Soils and waves: particulate materials behavior, characterization and process monitoring," Journal of Soils and Sediments, vol. 1, no. 2, p. 130, 2001.

[3] M. Meng and F. Wang, "Theoretical analyses and experimental research on a cement concrete dielectric model," Journal of Materials in Civil Engineering, vol. 25, no. 12, pp. 1959-1963, 2013.

[4] S. Lambot, I. Van den Bosch, B. Stockbroeckx, P. Druyts, M. Vanclooster, and E. Slob, "Frequency dependence of the soil electromagnetic properties derived from ground-penetrating 
radar signal inversion," Subsurface Sensing Technologies and Applications, vol. 6, no. 1, pp. 73-87, 2005.

[5] M. I. Mishchenko and L. Liu, "Electromagnetic scattering by densely packed particulate ice at radar wavelengths: exact theoretical results and remote-sensing implications," Applied Optics, vol. 48, no. 13, pp. 2421-2426, 2009.

[6] A. M. Nicolson and G. F. Ross, "Measurement of the intrinsic properties of materials by time-domain techniques," IEEE Transactions on Instrumentation and Measurement, vol. 19, no. 4, pp. 377-382, 1970.

[7] Y.-J. Cheng, Z.-X. Xia, L. Wang, and Y. Fan, "Frequency reconfigurable microwave reaction experiment apparatus for coal desulphurization," Journal of the University of Electronic Science and Technology of China, vol. 43, no. 1, pp. 31-35, 2014.

[8] Y. J. Cheng and X. L. Liu, "W-band characterizations of printed circuit board based on substrate integrated waveguide multiresonator method," IEEE Transactions on Microwave Theory and Techniques, vol. 64, no. 2, pp. 599-606, 2016.

[9] D. A. Robinson and S. P. Friedman, "The effective permittivity of dense packings of glass beads, quartz sand and their mixtures immersed in different dielectric backgrounds," Journal of NonCrystalline Solids, vol. 305, no. 1-3, pp. 261-267, 2002.

[10] A. M. Shutko and E. M. Reutov, "Mixture formulas applied in estimation of dielectric and radiative characteristics of soils and grounds at microwave frequencies," IEEE Transactions on Geoscience and Remote Sensing, vol. 20, no. 1, pp. 29-32, 1982.

[11] G. Y. Chernyak and H. Heimann, Dielectric Methods for Investigating Moist Soils, Israel Program for Scientific Translations, Jerusalem, Israel, 1967.

[12] G. C. Topp, J. L. Davis, and A. P. Annan, "Electromagnetic determination of soil water content: measurements in coaxial transmission lines," Water Resources Research, vol. 16, no. 3, pp. 574-582, 1980.

[13] D. A. Robinson and S. P. Friedman, "Electrical conductivity and dielectric permittivity of sphere packings: measurements and modelling of cubic lattices, randomly packed monosize spheres and multi-size mixtures," Physica A: Statistical Mechanics and its Applications, vol. 358, no. 2-4, pp. 447-465, 2005.

[14] Z. X. Xia, Y. J. Cheng, and Y. Fan, "Frequency-reconfigurable TM010-mode reentrant cylindrical cavity for microwave material processing," Journal of Electromagnetic Waves and Applications, vol. 27, no. 5, pp. 605-614, 2013.

[15] B. Sareni, L. Krähenbühl, A. Beroual, and C. Brosseau, "Effective dielectric constant of periodic composite materials," Journal of Applied Physics, vol. 80, no. 3, pp. 1688-1696, 1996.

[16] B. Sareni, L. Krähenbühl, A. Beroual, and C. Brosseau, "Effective dielectric constant of random composite materials," Journal of Applied Physics, vol. 81, no. 5, pp. 2375-2383, 1997.

[17] K. K. Karkkainen, A. H. Sihvola, and K. I. Nikoskinen, "Effective permittivity of mixtures: numerical validation by the FDTD method," IEEE Transactions on Geoscience and Remote Sensing, vol. 38, no. 3, pp. 1303-1308, 2000.

[18] M. Suzuki and T. Oshima, "Verification of a model for estimating the void fraction in a three-component randomly packed bed," Powder Technology, vol. 43, no. 2, pp. 147-153, 1985.

[19] L. Oger, J. P. Troadec, D. Bideau, J. A. Dodds, and M. J. Powell, "Properties of disordered sphere packings I. Geometric structure: statistical model, numerical simulations and experimental results," Powder Technology, vol. 46, no. 2-3, pp. 121-131, 1986.

[20] P. R. Rios, "Comparison between a computer simulated and an analytical grain size distribution," Scripta Materialia, vol. 40, no. 6, pp. 665-668, 1999.
[21] R. Al-Raoush and M. Alsaleh, "Simulation of random packing of polydisperse particles," Powder Technology, vol. 176, no. 1, pp. 47-55, 2007.

[22] M. Elimelech, J. Gregory, and X. Jia, Particle Deposition and Aggregation: Measurement, Modelling and Simulation, Butterworth-Heinemann, 2013.

[23] D. He, N. N. Ekere, and L. Cai, "Computer simulation of random packing of unequal particles," Physical Review E, vol. 60, no. 6, pp. 7098-7104, 1999.

[24] G. D. Scott, "Packing of spheres: packing of equal spheres," Nature, vol. 188, no. 4754, pp. 908-909, 1960.

[25] Y. Konakawa and K. Ishizaki, "The particle size distribution for the highest relative density in a compacted body," Powder Technology, vol. 63, no. 3, pp. 241-246, 1990.

[26] J. Rodríguez, C. H. Allibert, and J. M. Chaix, "A computer method for random packing of spheres of unequal size," Powder Technology, vol. 47, no. 1, pp. 25-33, 1986.

[27] L. D. Landau, J. Bell, M. Kearsley, L. Pitaevskii, E. Lifshitz, and J. Sykes, Electrodynamics of Continuous Media, Elsevier, Philadelphia, Pa, USA, 1984.

[28] J. A. Reynolds and J. M. Hough, "Formulae for dielectric constant of mixtures," Proceedings of the Physical Society, Section B, vol. 70, no. 8, pp. 769-775, 1957.

[29] J. C. Maxwell-Garnett, "Colours in metal glasses and in metallic films," Philosophical Transactions of the Royal Society of London, vol. 203, no. 359-371, pp. 385-420, 1904.

[30] Ć. Blanchard, J. A. Portí, J. A. Morente, A. Salinas, and E. A. Navarro, "Determination of the effective permittivity of dielectric mixtures with the transmission line matrix method," Journal of Applied Physics, vol. 102, no. 6, Article ID 064101, 2007.

[31] C. J. F. Böttcher, O. C. van Belle, P. Bordewijk, and A. Rip, Theory of Electric Polarization, Elsevier Science, Philadelphia, Pa, USA, 1978.

[32] W. B. Weir, "Automatic measurement of complex dielectric constant and permeability at microwave frequencies," Proceedings of the IEEE, vol. 62, no. 1, pp. 33-36, 1974. 


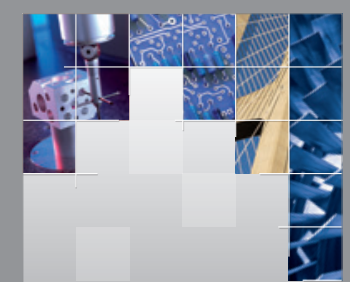

\section{Enfincering}
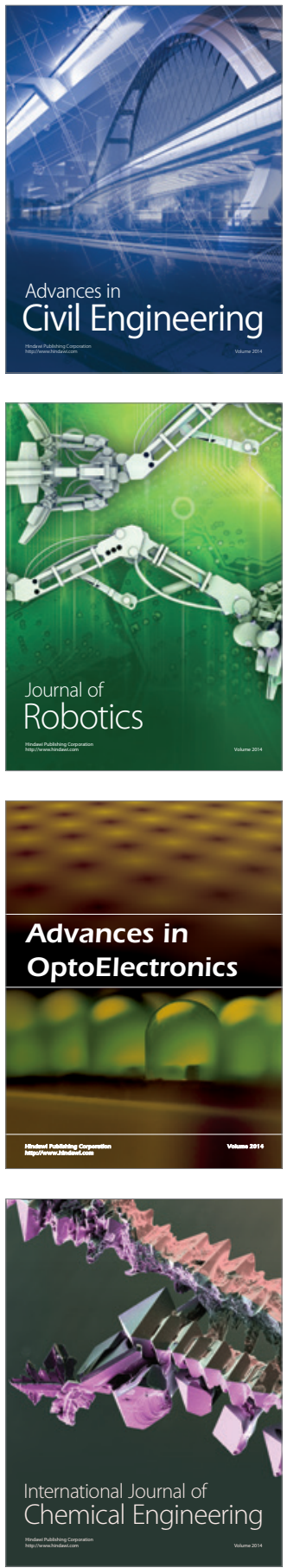

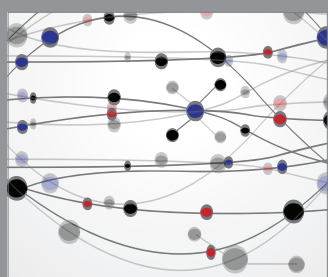

The Scientific World Journal

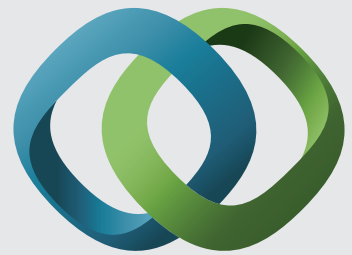

\section{Hindawi}

Submit your manuscripts at

http://www.hindawi.com
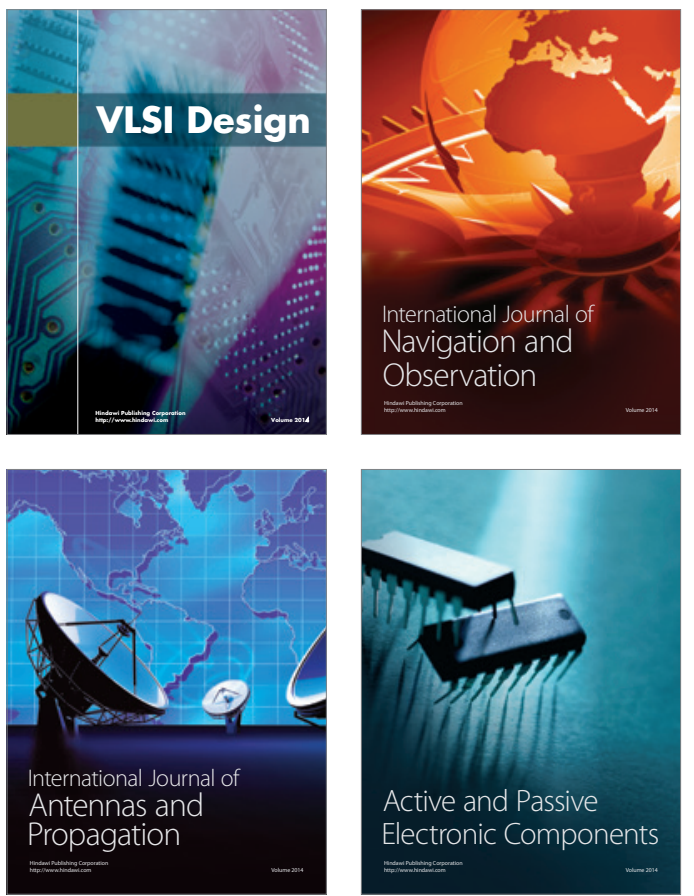
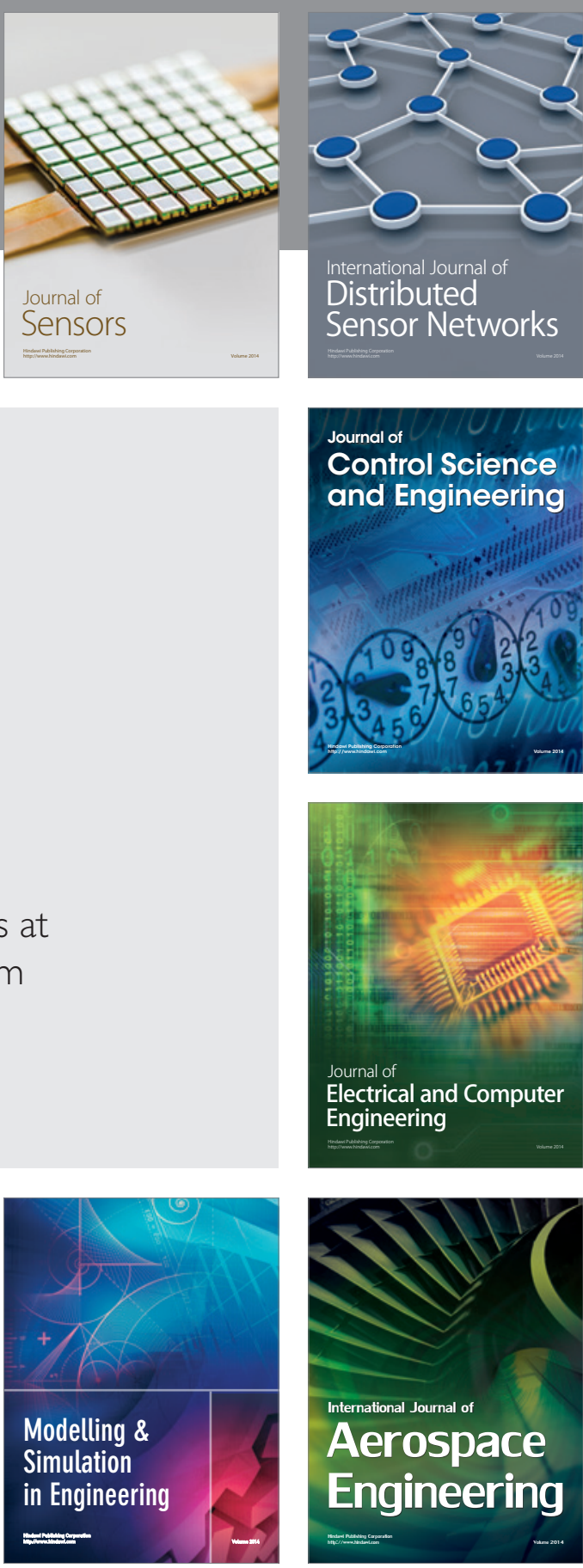

International Journal of

Distributed

Sensor Networks

Journal of

Control Science

and Engineering
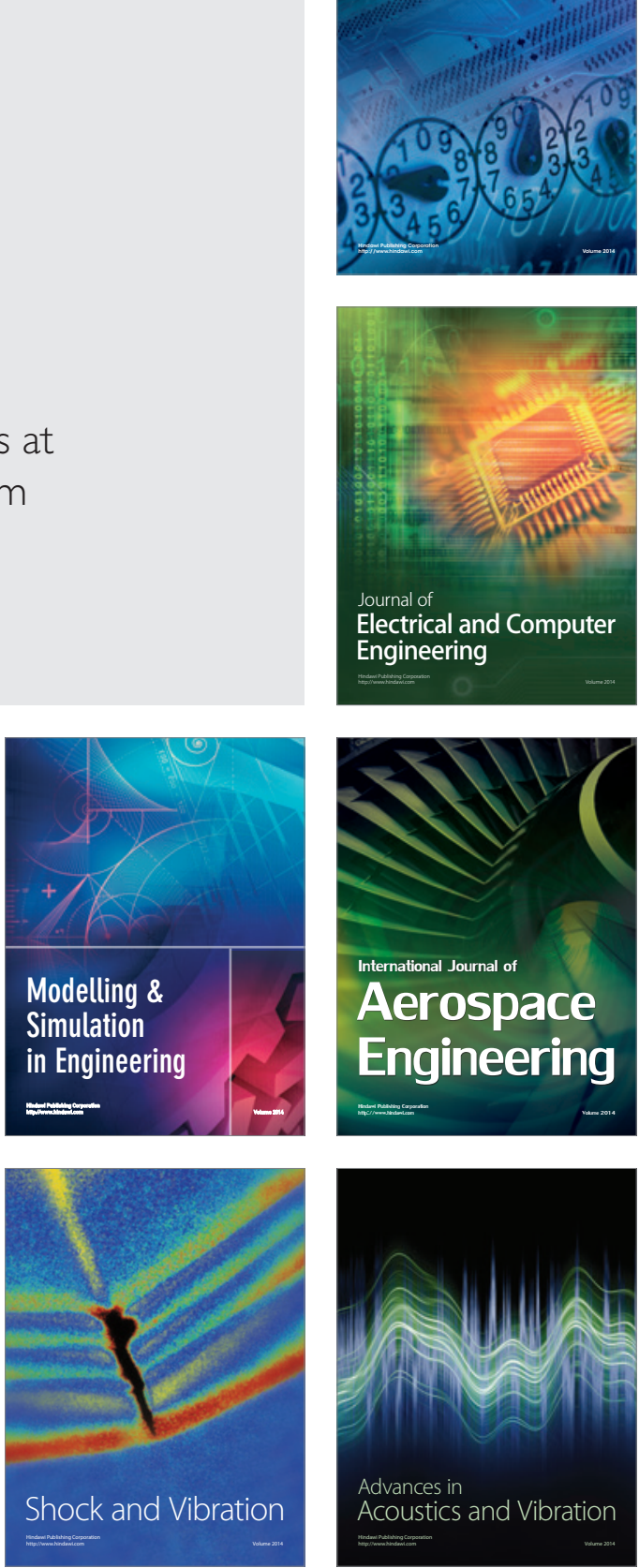